Georgian Mathematical Journal

Volume 13 (2006), Number 1, 7-10

\title{
HOLOMORPHIC VECTOR BUNDLES ON HOLOMORPHICALLY CONVEX COMPLEX MANIFOLDS
}

\author{
EDOARDO BALLICO
}

\begin{abstract}
Let $X$ be a holomorphically convex complex manifold and $\operatorname{Exc}(X) \subseteq X$ the union of all positive dimensional compact analytic subsets of $X$. We assume that $\operatorname{Exc}(X) \neq X$ and $X$ is not a Stein manifold. Here we prove the existence of a holomorphic vector bundle $E$ on $X$ such that $(E \mid U) \oplus \mathcal{O}_{U}^{m}$ is not holomorphically trivial for every open neighborhood $U$ of $\operatorname{Exc}(X)$ and every integer $m \geq 0$. Furthermore, we study the existence of holomorphic vector bundles on such a neighborhood $U$, which are not extendable across a 2-concave point of $\partial(U)$.
\end{abstract}

2000 Mathematics Subject Classification: 32L05, 32E05, 32F10.

Key words and phrases: Holomorphic vector bundle, holomorphically convex complex manifold, Stein space, $q$-concave complex space.

\section{INTRODUCTION}

A famous theorem of Grauert states that on a complex Stein space the holomorphic and the topological classification of vector bundles are the same. In particular every holomorphic vector bundle on a one-dimensional or a contractible Stein space is holomorphically trivial. A suitable extension of Grauert's theorem to 0-convex complex manifolds was proved by G. Henkin and J. Leiterer (see [5] and [3]). J. Winkelmann proved that on any $n$-dimensional compact complex manifold there is a non-trivial holomorphic vector bundle of rank at most $n$ ([6] and [7], Theorem 7.13.1). Let $X$ be a connected holomorphically convex complex manifold and $f: X \rightarrow Z$ be its Remert reduction $f: X \rightarrow Z$. We recall that $f$ is proper, $Z$ is a Stein space, $f_{*}\left(\mathcal{O}_{X}\right)=\mathcal{O}_{Z}$ and that the pair $(Z, f)$ is uniquely determined by these properties. Furthermore, $f$ is surjective and for any $P \in X$ the fiber $f^{-1}(f(P))$ is the union of all irreducible compact analytic subsets of $X$ containing $P$. $X$ is Stein if and only if $f$ is an isomorphism. $X$ is compact if and only if $Z$ is a point. Let $\operatorname{Exc}(X):=\operatorname{Exc}(f):=\{P \in X: f$ is not a local isomorphism at $P\}$ be the exceptional locus of $f$. $\operatorname{Exc}(f)$ is the union of all the positive dimensional irreducible compact analytic subsets of $X$. We will also call it the exceptional subset of $X \cdot \operatorname{Exc}(X)=X$ if and only if $\operatorname{dim}(Z)<\operatorname{dim}(X)$. In this paper we will only consider the case $\operatorname{dim}(X)=\operatorname{dim}(Z)$. For the case $1 \leq \operatorname{dim}(X)-\operatorname{dim}(Z) \leq 2$, see [2], Theorem 1.2. In Section 2 we will prove the following two theorems.

Theorem 1. Let $X$ be a holomorphically convex complex manifold such that $\operatorname{Exc}(X) \neq X$ and $\operatorname{Exc}(X)$ contains a hypersurface of $X$. Then there is a 
holomorphic line bundle $L$ on $X$ such that $(L \mid U) \oplus \mathcal{O}_{U}^{m}$ is not holomorphically trivial for every open neighborhood $U$ of $\operatorname{Exc}(X)$ and every integer $m \geq 0$.

Theorem 2. Let $X$ be a holomorphically convex complex manifold such that $\operatorname{Exc}(X) \neq X$ and $\operatorname{Exc}(X)$ contains no hypersurface of $X$. Then the tangent bundle $T X$ is not holomorphically trivial. Furthermore, for every open neighborhood $U$ of $\operatorname{Exc}(X)$ and every integer $m \geq 0$ on $U$ the vector bundle $T X \mid U \oplus \mathcal{O}_{U}^{\oplus m}$ is not holomorphically trivial.

From Theorems 2 and 1 we obviously obtain the following result.

Corollary 1. Let $X$ be a holomorphically convex complex manifold such that $\operatorname{Exc}(X) \neq X$. Then there exists a holomorphic vector bundle $E$ on $X$ such that $\operatorname{rank}(E) \leq \operatorname{dim}(X)$ and for every open neighborhood $U$ of $\operatorname{Exc}(X)$ and every integer $m \geq 0$ the holomorphic vector bundle $E \mid U \oplus \mathcal{O}_{U}^{\oplus m}$ is not holomorphically trivial.

For the results corresponding to Corollary 1 when $X$ is 0 -convex, i.e. when $f(\operatorname{Exc}(X))$ is a finite set, see [2], Theorem 1.2.

Now we will drop the assumption $\operatorname{Exc}(X) \neq X$ and consider the problem of the existence of non-trivial holomorphic vector bundles on certain open subsets of $X$. We will obtain a very easy extension of [1] to this set-up. Let $U \subseteq X$ be an open subset of $X$. We will say that $U$ is $f$-saturated if $U=f^{-1}(f(U))$. Since $f$ is proper and surjective, the set $f(U)$ is open in $Z$ for every $f$-saturated open subset of $X$. Let $X$ be a complex space and $U \subset X$ be an open subset of $X, E$ a holomorphic vector bundle on $U$ and $P \in \partial(U)$. We will say that $E$ extends across $P$ if there are an open neighborhood $W$ of $P$ in $X$ and a holomorphic vector bundle $F$ on $W$ such that $F|U \cap W \cong E| U \cap W$. Since a holomorphic vector bundle is locally trivial, by restricting, if necessary, $W$ we may assume that $F$ is trivial. Hence $E$ extends across $P$ if and only if there is an open neighborhood $A$ of $P$ in $X$ such that $E \mid U \cap A$ is trivial.

Proposition 1. Let $X$ be an irreducible holomorphically convex complex space such that $\operatorname{Exc}(X) \neq X$ and its Remmert reduction $f: X \rightarrow Z$ has the property that $Z$ is biholomorphic open subset of $\mathbb{C}^{n}, n \geq 3$. Let $U$ be an open subset of $X$ containing $\operatorname{Exc}(X)$ and $P \in \partial(U)$ such that the domain $f(U)$ is 2 -concave at $f(P)$. Then there is a holomorphic vector bundle $E$ on $U$ such that $\operatorname{rank}(E)=n-1, E$ does not extend across $P$, but it extends across every other point of $\partial(U)$.

Proof. By [1], Theorem 1.1, there is a holomorphic vector bundle $F$ on $f(U)$ such that $F$ does not extend across $f(P)$, but it extends across all the other boundary points of $f(U)$ (seen as an open subset of $\mathbb{C}$ ). In particular $F$ extends along all the boundary points of $f(U)$ inside $Z$, except $f(P)$. The proof of [1], Theorem 1.1, shows that there is such a vector bundle $F$ with the additional property $\operatorname{rank}(F)=n-1$. Set $E:=f^{*}(E)$. $E$ is a rank $n-1$ holomorphic vector bundle on $U$. Since $\operatorname{Exc}(X) \subset X$ and $P \notin U, f$ is biholomorphic in a neighborhood of $P$ and hence $f^{*}(E)$ does not extend across $P$. Similarly (but 
this part is true even for more simpler reasons), $f$ extends across all other points of $\partial(U)$.

Remark 1. Take the set-up of Proposition 1 and its proof, but drop the assumption " $\operatorname{Exc}(X) \subset U$ ". Assume only that $U$ is $f$-saturated. The properness of $f$, the fact that $f_{*}\left(\mathcal{O}_{X}\right)=\mathcal{O}_{Z}$ and the proof of Proposition 1 show that there is no $f$-saturated open subset $W$ of $P$ in $X$ such that $f^{*}(F) \mid W \cap U$ is trivial.

By the proofs of Proposition 1, Remark 1 and [1], Theorem 1.2, we immediately get the following result.

Proposition 2. Let $X$ be a holomorphically convex complex space, $f: X \rightarrow Z$ its Remmert reduction, $U$ an open $f$-saturated subset of $X$ and $P \in \partial(U)$ such that $f(U)$ is 2-concave at $f(P)$ and $Z$ has dimension at least three at $P$. Then there exists a holomorphic vector bundle $E$ on $U$ such that $E$ extends across every point of $\partial(U) \backslash\{P\}$, but there is no $f$-saturated open neighborhood $W$ of $f^{-1}(f(P))$ in $X$ such that $E \mid U \cap W$ is trivial. If $\operatorname{Exc}(X) \subset U$, then $E$ does not extend across $P$.

\section{THE PROOFS}

Proof of Theorem 1. By assumption, there is an irreducible component $D$ of $\operatorname{Exc}(X)$ which is a closed hypersurface of $X$. Since $X$ is smooth, the sheaf $\mathcal{I}_{D, X}$ is a holomorphic line bundle, $L$, on $X$. Fix a neighborhood $U$ of $\operatorname{Exc}(X)$ and an integer $m \geq 0$ and assume $(L \mid U) \oplus \mathcal{O}_{U}^{\oplus m}$ trivial. Since $L \mid U$ is the determinant of $(L \mid U) \oplus \mathcal{O}_{U}^{\oplus m}, L \mid U$ must be trivial. Hence its dual $L^{*} \mid U$ is trivial. Hence there is a holomorphic function $g$ on $U$ whose zero-locus is scheme-theoretically exactly $D$. Since $\operatorname{Exc}(U) \subset U$ and $f_{*}\left(\mathcal{O}_{X}\right)=\mathcal{O}_{Z}, f(U)$ is an open subset of $Z$ and there is a holomorphic function $h$ on $f(U)$ such that $g=h \circ f$. Since $X$ is normal, $Z$ is normal and in particular every local ring $\mathcal{O}_{Z, Q}, Q \in Z$, is an integral domain. We have $f(D)=\{h=0\}$. Notice that $\{h=0\}$ is an effective Cartier divisor of $Z$ and hence it has pure codimension one in $Z$ at each of its points. Since $D \subseteq \operatorname{Exc}(X)$, we have $\operatorname{dim}(f(D))<\operatorname{dim}(D)=\operatorname{dim}(X)-1=\operatorname{dim}(Z)-1$, a contradiction.

Proof of Theorem 2. Fix an integer $m \geq 0$ and assume the existence of an open neighborhood $U$ of $\operatorname{Exc}(X)$ such that $T U \oplus \mathcal{O}_{U}^{\oplus m}$ is trivial. Since $U \backslash \operatorname{Exc}(X) \cong f(U) \backslash f(\operatorname{Exc}(X))$, the restriction to $f(U) \backslash f(\operatorname{Exc}(X))$ of $\Theta_{f(U)} \oplus$ $\mathcal{O}_{f(U)}^{\oplus m}$ is trivial, where $\Theta_{f(U)}$ denotes the tangent sheaf of $f(U)$. By its very definition $\Theta_{f(U)}$ is the dual of the cotangent sheaf of $f(U)$ and in particular it is isomorphic to a dual of a coherent analytic sheaf with $\operatorname{rank} \operatorname{dim}(Z)$ at each smooth point of $f(U)$. In particular $\Theta_{f(U)}$ is the so-called reflexive sheaf. Since $X$ is smooth, it is normal. Hence the universal properties of the normalization and of the Remmert reduction imply that $Z$ is normal. On a normal complex space any reflexive sheaf is uniquely determined by its restriction to an open subset whose complementary is a closed analytic subset with codimension at least two. Since the trivial vector bundle $\mathcal{O}_{f(U)}^{\oplus \operatorname{dim}(X)+m}$ is an extension of $\Theta_{f(U)} \oplus$ $\mathcal{O}_{f(U)}^{\oplus m} \mid f(U) \backslash f(\operatorname{Exc}(X)), \Theta_{f(U)} \oplus \mathcal{O}_{f(U)}^{\oplus m}$ is trivial. Hence $\Theta_{f(U)}$ is locally free. 
Since $\operatorname{dim}(f(\operatorname{Exc}(X)))<\operatorname{dim}(\operatorname{Exc}(X)) \leq \operatorname{dim}(X)-2, f(U)$ is smooth ([4], Corollary at p. 318). The holomorphic map $f: U \rightarrow f(U)$ is a holomorphic map between smooth manifolds which is an isomorphism outside a closed analytic subset of $U$ with codimension at least two. This implies that $f$ is an isomorphism (use the determinant of the differential $d f: T X \rightarrow f^{*}(T Z)$ ). By the universal property of the Remmert reduction, this implies $X$ is Stein, a contradiction.

\section{ACKNOWLEDGEMENT}

The author was partially supported by MIUR and GNSAGA of INdAM (Italy).

\section{REFERENCES}

1. E. BALlico, Extension of holomorphic vector bundles across a boundary point. Complex Var. Theory Appl. 48(2003), No. 11, 987-989.

2. E. BALLICO, Holomorphic vector bundles on certain holomorphically convex complex manifolds. Boll. Unione Mat. Ital. (to appear).

3. M. ColţoiU, On the Oka-Grauert principle for 1-convex manifolds. Math. Ann. 310(1998), No. 3, 561-569.

4. H. Flenner, Extendability of differential forms on nonisolated singularities. Invent. Math. 94(1988), No. 2, 317-326.

5. G. Henkin and J. Leiterer, The Oka-Grauert principle without induction over the base dimension. Math. Ann. 311(1998), No. 1, 71-93.

6. J. Winkelmann, Every compact complex manifold admits a holomorphic vector bundle. Rev. Roumaine Math. Pures Appl. 38(1993), No. 7-8, 743-744.

7. J. Winkelmann, Complex analytic geometry of complex parallelizable manifolds. Mém. Soc. Math. Fr. (N.S.) No. $72-73$ (1998), 219 pp.

(Received 17.04.2004)

Author's address:

Dept. of Mathematics

University of Trento

38050 Povo (TN), Italy

E-mail: ballico@science.unitn.it 